\title{
Heavy element abundances in AGB stars. II. 47 Tucanae
}

\author{
Wylie, E.C. ${ }^{1}$, Cottrell, P.L. ${ }^{1}$, and Sneden, C.A. ${ }^{2}$ \\ ${ }^{1}$ University of Canterbury, New Zealand \\ email: e.wylie@phys.canterbury.ac.nz \\ ${ }^{2}$ University of Texas, USA
}

\begin{abstract}
This research forms the second part of an investigation into heavy element abundances in Asymptotic Giant Branch (AGB) stars in different stellar environments. High resolution spectroscopy was taken of seven known AGB stars in 47 Tucanae and spectrum synthesis undertaken to obtain abundances for s-process elements. Contrary to theoretical predictions, preliminary results show that these AGB stars do show s-process enhancements relative to previously studied Red Giant Branch (RGB) stars in the same cluster.
\end{abstract}

Keywords. Nucleosynthesis, abundances, techniques: spectroscopic, stars: AGB

\section{Summary}

Present AGB theory predicts that the AGB stars in 47 Tuc are too low in mass to undergo third dredge-up and consequently should not show s-process element enhancements over their RGB progenitors (Brown \& Wallerstein, 1992). However, our new study, see Table 1, suggests that an s-process enhancement is present relative to the RGB stars in the same globular cluster. Several checks were undertaken to ensure this enhancement was genuine and not a product of discrepancies between the two studies.

Differences in transition probabilities are the most likely cause of the abundance disagreements. Brown \& Wallerstein adopted a combination of laboratory values and values obtained from a reverse solar analysis. All log gf values used for this research were obtained via the latter method. Any disagreements between the current and previous log gf values are not sufficiently large to affect the abundance. As an additional test, spectrum synthesis of several lighter elements was undertaken to ensure that the abundances observed in the AGB stars matched those observed in the RGB stars. Results suggest that for all stars, the lighter elements agree to within \pm 0.10 dex. This general agreement in lighter element abundances suggests the discrepancies seen in the heavier elements can not be explained by differences in modelling or synthesis techniques.

Perhaps the most convincing check was to use the abundances obtained for the RGB stars to compute a synthetic AGB spectrum and compare it to an observed spectrum of an AGB star, see Figure 1. While atmospheric parameters obviously differ between the two types of stars, the difference between the RGB Zr abundances and the AGB observed

Table 1. Element abundances in RGB and AGB stars of 47 Tuc. In order they are: FeI, FeII, YI, YII, ZrI, ZrII, LaII, NdII and EuII.

\begin{tabular}{c|ccccccccc}
\hline Species & 26 & 26.1 & 39 & 39.1 & 40 & 40.1 & 57.1 & 60.1 & 63.1 \\
\hline RGB - $\log \epsilon$ & 6.8 & 6.9 & 1.7 & 2.1 & 1.5 & & 0.6 & & 0.2 \\
AGB - $\log \epsilon$ & 7.0 & 7.0 & 2.3 & 2.3 & 2.7 & 2.6 & 1.0 & 1.4 & 0.1 \\
\hline
\end{tabular}




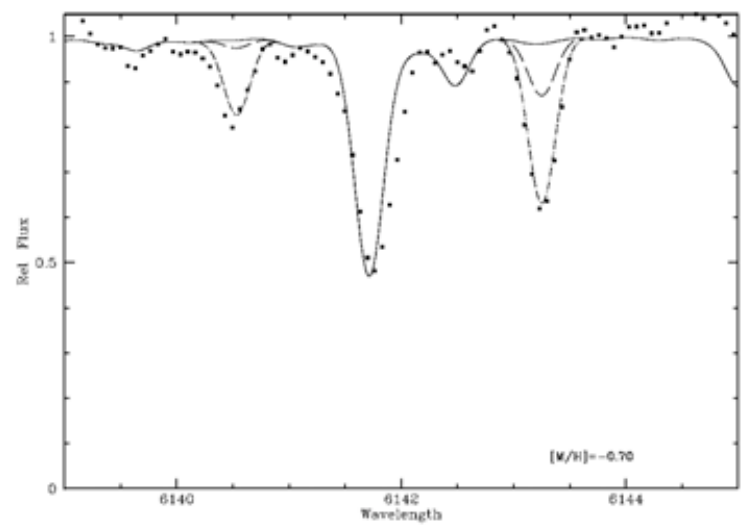

Figure 1. The Zr abundance obtained for RGB stars compared to the observed spectrum of an AGB star. The long dashed line shows the $\mathrm{Zr}$ abundance in the RGB stars and the observed spectrum of the AGB stars is shown by dots. The solid line represents no $\mathrm{Zr}$ present, while the short dashed line is the $\mathrm{Zr}$ abundance derived for the AGB stars.

spectrum is too large to be explained by relatively small changes in temperature, gravity or microturbulence.

An alternative cause for the apparent s-process enhancement is the effect caused by ionisation equilbrium, which would cause imbalances between neutral and ionised species. In the current study both neutral and ionised lines were used to derive abundances for $\mathrm{Y}$ and $\mathrm{Zr}$. As is shown in Table 1, there is excellent agreement between abundances obtained from neutral and ionised lines in the AGB stars, with the difference never more than 0.1 dex. Consequently, ionisation equilbrium effects are not a cause for this observed s-process enhancement.

Elevated s-process abundances in stars can come from two possible physical sources: extrinsic pollution or internal processes. The possibility of extrinsic pollution in this case seems remote. There is no model that suggests spatial selection for extrinsic pollution. Consequently, all stars of the globular cluster should be polluted, and therefore enhanced, in the same way. It is unlikely that AGB stars should show enhancement from extrinsic pollution while the RGB stars do not.

This leaves perhaps only one strong possibility - that the AGB stars are showing sprocess enhancement due to some intrinsic process. The most likely method to enhance s-process elements is the third dredge-up phase which stars undergo while on the AGB. If third dredge-up is present, the star should also show an enhancement of $\mathrm{C}$, which increases as the star progresses through the recurring dredge-ups. The carbon content of these AGB stars is currently being studied using synthesis of $\mathrm{C}_{2}$ in a region shown to be sensitive to $\mathrm{C}$ abundance (Cottrell and Sneden 1986). Preliminary results show that $\mathrm{C}$ is slightly enhanced in these stars. This observation lends support to the theory that these stars, contradictory to present AGB modelling, are undergoing a third dredge-up stage.

More detailed results and discussion will be available in the research paper to follow (Wylie, Cottrell, Sneden \& Lattanzio, in preparation).

\section{References}

Brown, J. \& Wallerstein, G. 1992, AJ 104, 1818

Cottrell, P.L. \& Sneden C.A. 1986, A\&广A 161, 314 


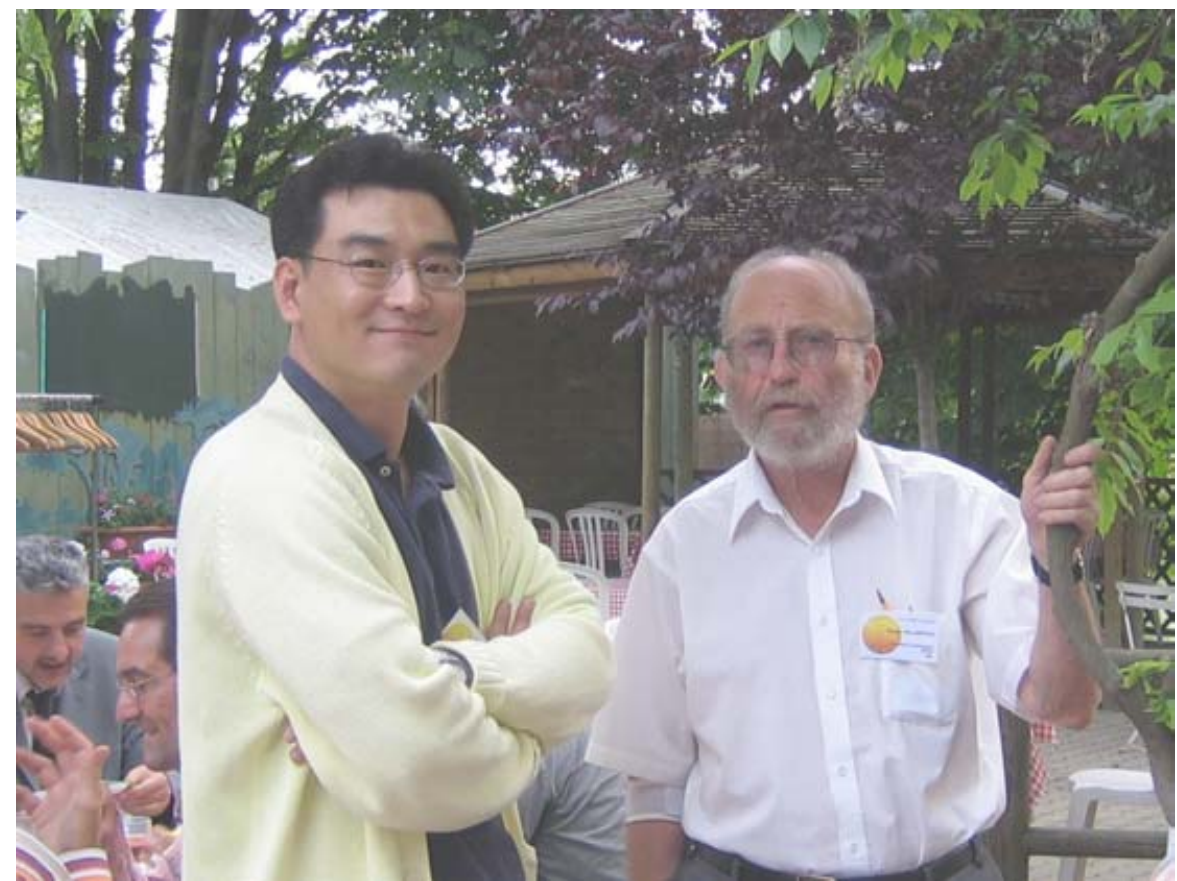

Jaewoo Lee and George Wallerstein at the conference dinner.

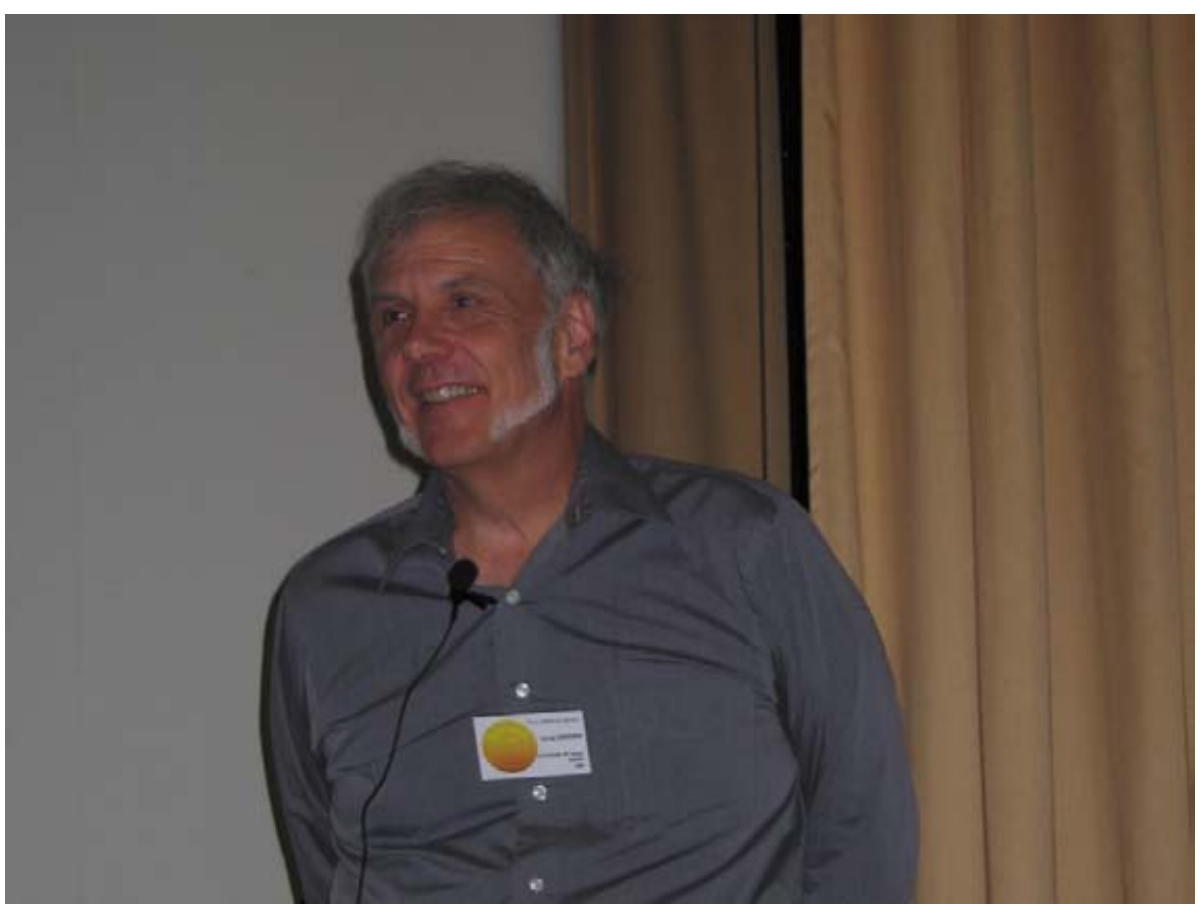

Chris Sneden during his talk on the Globular Cluster - field connection. 


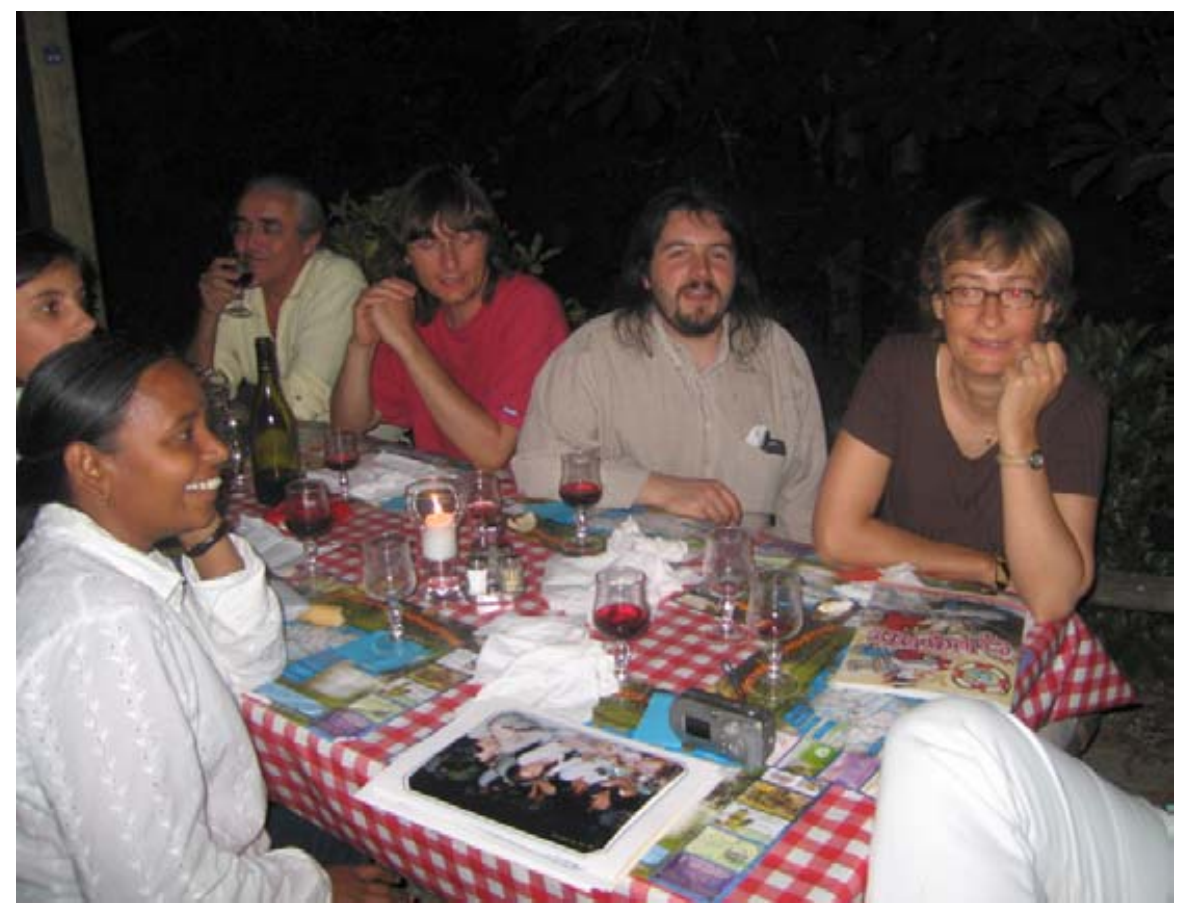

At the conference dinner, Alejandra Recio Blanco and Thirupathi Sivarani on one side of the table facing, from left to right, José Renan de Medeiros, Patrick de Laverny, Thomas Masseron and Agnès Lèbre.

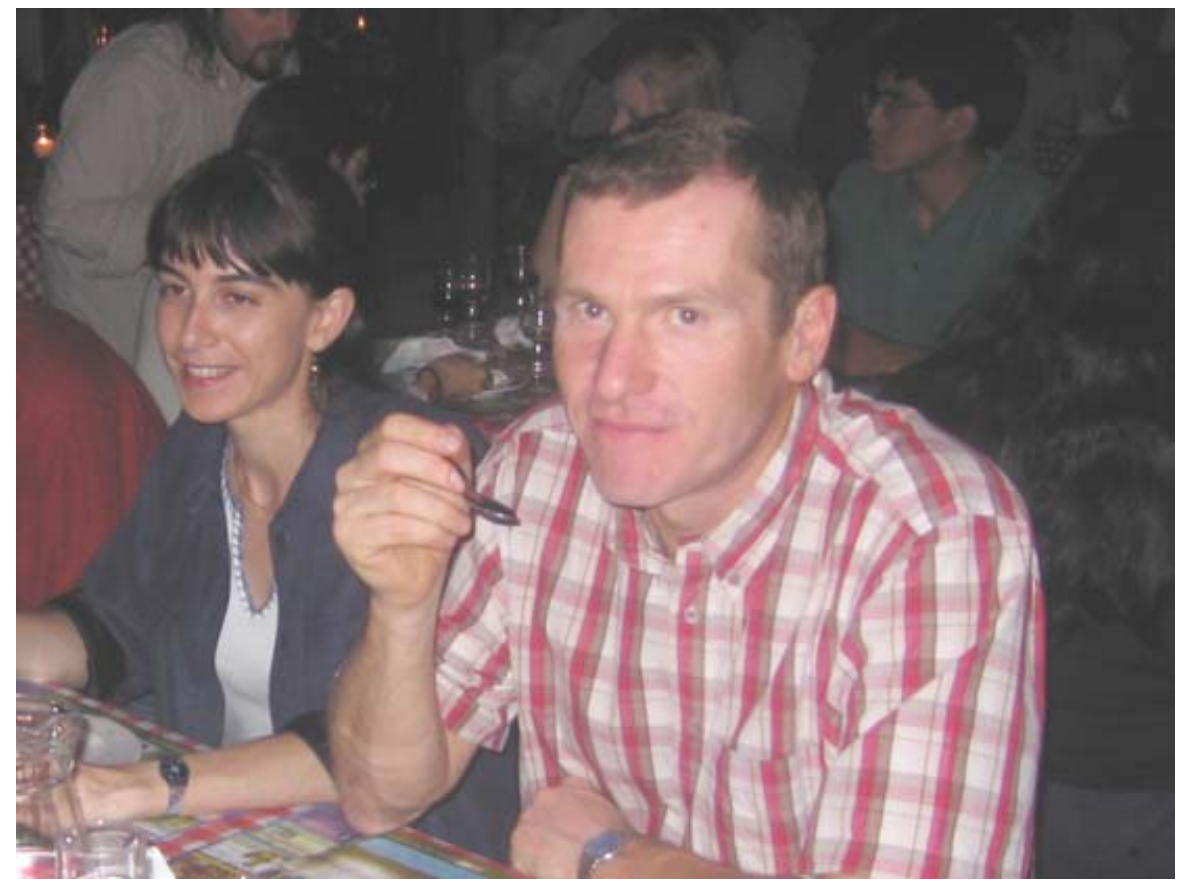

Bertrand Plez and Sophie van Eck at the conference dinner. 